\title{
0 processo de implementação do diagnóstico de enfermagem no Hospital Universitário da Universidade de São Paulo
}

\author{
THE NURSING DIAGNOSIS IMPLEMENTATION PROCESS AT THE UNIVERSITY \\ HOSPITAL OF THE UNIVERSITY OF SÃO PAULO
}

\author{
EL PROCESO DE IMPLEMENTAR EL DIAGNÓSTICO DE ENFERMERÍA EN EL \\ HOSPITAL UNIVERSITARIO DE LA UNIVERSIDAD DE SAO PAULO
}

\section{Antônio Fernandes Costa Lima ${ }^{1}$, Paulina Kurcgant ${ }^{2}$}

\section{RESUMO}

Há mais de duas décadas, o Departamento de Enfermagem (DE) do Hospital Universitário da Universidade de São Paulo (HU-USP) implementou o modelo assistencial, denominado Sistema de Assistência de Enfermagem (SAE), que integra três fases: o Histórico, a Evolução e a Prescrição de Enfermagem e que vem sendo desenvolvido pelos enfermeiros do DE como um instrumento norteador da assistência, do ensino e da pesquisa. Tendo em vista a informatização do SAE, os enfermeiros iniciaram discussões acerca da necessidade de mudanças que agilizassem o processo de trabalho com a proposição da implementação do Diagnóstico de Enfermagem, como mais uma etapa do SAE, e com a revisão das condutas/intervenções de enfermagem. Para tanto, tornou-se imprescindível a adoção de um sistema padronizado de linguagem do processo assistencial a fim de que se pudesse realizar a captura, agrupamento e classificação dos dados para análise e transformação em informações. O presente estudo tem como objetivo compartilhar com outros enfermeiros essa experiência no processo de implementação do Diagnóstico de Enfermagem como segunda etapa do SAE.

\section{DESCRITORES}

Processos de enfermagem. Diagnóstico de enfermagem. Inovação organizacional.

\section{ABSTRACT}

More than two decades ago the Nursing Department at the University Hospital of the University of São Paulo implemented the care model called Nursing Care System (NCS), which is comprised of three phases: Background, Evaluation and Nursing Prescription. Since then, it is being developed by the Nursing Department nurses as a guiding instrument for care, teaching and research. Having in mind the automation of the NCS, the nurses began to discuss the need for changes that could speed up the work process with the proposal of implementation of the Nursing Diagnosis as another step of the NCS and with the review of nursing action/interventions. In order to do so, the adoption of a standardized system of the care process language became essential so as to make possible data capture, grouping and classification for analysis and their transformation into information. The present study aims at sharing with other nurses this experience on the implementation process of the Nursing Diagnosis as a second step of the NCS. nd step of the NCS.

\section{KEY WORDS}

Nursing process.

Nursing diagnosis.

Organizational innovation.

\section{RESUMEN}

Hace más de dos décadas, el Departamento de Enfermería (DE) del Hospital Universitario de la Universidad de São Paulo (HUUSP) implementó el modelo asistencial, denominado Sistema de Asistencia de Enfermería(SAE), que integra tres fases: el Histórico, la Evolución y la Prescripción de Enfermería y que viene siendo desarrollado por los enfermeros del DEcomo un instrumento orientador de la asistencia, de la enseñanza y de la investigación. Buscando informarse sobre el SAE, los enfermeros iniciaron discusiones acerca de la necesidad de cambios que agilicen el proceso de trabajo con la proposición de la implementación delDiagnóstico de Enfermería, como una etapa más del SAE, y con la revisión de las conductas/intervenciones de enfermería. Para tal efecto, se tornó imprescindible la adopción de un sistema patronizado de lenguaje del proceso asistencial a fin de que se pueda realizar la captura, agrupación y clasificación de los datos para el análisis y transformación en informaciones. El presente estudio tiene como objetivo compartir con otros enfermeros esa experiencia en el proceso de implementación del Diagnóstico de Enfermería como segunda etapa del SAE.

\section{DESCRIPTORES}

Procesos de enfermería.

Diagnóstico de enfermería. Innovación organizacional.

\footnotetext{
* Extraído da Tese:

"Significados que as enfermeiras assistenciais de um hospital universitário atribuem ao processo de implementação do diagnóstico de enfermagem como etapa do

Sistema de Assistência de Enfermagem - SAE", Escola de Enfermagem da Universidade de São Paulo (EEUSP), 2004.

1 Enfermeiro - Diretor Técnico do Serviço de Apoio Educacional do Hospital Universitário da USP. Mestre em Fundamentos de Enfermagem pela EEUSP. Doutor pelo Programa Interunidades da USP. tonifer@usp.br

2 Enfermeira - Professora Titular do Departamento de Orientação Profissional da EEUSP.

Docente da área de Administração em Enfermagem, pkurcg@usp.br
} 


\section{INTRODUÇÃO}

Há mais de duas décadas o Departamento de Enfermagem (DE) do Hospital Universitário da Universidade de São Paulo (HU-USP) fundamenta sua prática assistencial no processo de enfermagem, proposto por Horta ${ }^{(1)}$ e no conceito de Autocuidado de Orem ${ }^{(2)}$, que visa a satisfação das necessidades individuais e específicas do paciente/cliente e o desenvolvimento do potencial dos indivíduos para o autocuidado. Para a viabilização desta proposta foi implementado o modelo assistencial, denominado Sistema de Assistência de Enfermagem (SAE), composto por três fases: o Histórico, a Evolução e a Prescrição de Enfermagem, que vem sendo desenvolvido pelos enfermeiros do DE como um importante instrumento norteador da assistência, do ensino e da pesquisa.

Ao longo destes anos é inegável o sucesso e a eficácia deste modelo assistencial que tem a qualidade como elemento norteador. No entanto, os enfermeiros vêm apontando dificuldades que encontram no desenvolvimento desse modelo e a necessidade de mudanças que agilizem o processo de trabalho e otimizem, cada vez mais, a qualidade das suas ações cuidativas e educativas. Tendo em vista a informatização do SAE, como primeiro passo para essas transformações, acreditam ser necessária a revisão das condutas/intervenções de enfermagem.

Assim, para a informatização do SAE tornou-se imprescindível a utilização de um sistema padronizado de linguagem do processo assistencial a fim de que se pudesse realizar a captura de dados desse processo, para agrupá-los, classificá-los, analisá-los e transformá-los em informações. A padronização pressupõe a utilização de um conjunto de termos pré-estabelecidos que expressam: situações do processo saúde/doença que podem ser modificadas por intervenções de enfermagem; ações de enfermagem que modificam as situações do processo saúde/doença e resultados obtidos com a efetivação dessas ações. Outra necessidade considerada, na viabilização da padronização da linguagem, foi a implementação do diagnóstico de enfermagem como mais uma etapa do SAE.

O diagnóstico de enfermagem pode ser analisado sob várias perspectivas sendo, uma delas, a que o define como uma das etapas do processo de enfermagem ${ }^{(3)}$. Essa autora afirma que no contexto do processo de enfermagem, tradicionalmente descrito em cinco etapas: levantamento dos dados, diagnóstico, planejamento, implementação e avaliação, o diagnóstico é a conclusão do levantamento dos dados uma vez que o propósito primordial, desse levantamento, é a identificação das necessidades de cuidados de enfermagem a serem prestados aos pacientes.

Quando o processo de enfermagem é praticado como uma forma de investigação, conduz o enfermeiro no desenvolvimento de um estilo de pensamento que direciona os julgamentos na forma de diagnósticos de enfermagem, fornecendo base segura para a assistência de enfermagem ${ }^{(4)}$.

O desenvolvimento de um sistema de classificação para os diagnósticos de enfermagem, de forma científica e confiável, tem provado ser um processo lento e difícil. No entanto, apesar dos problemas, este sistema de classificação, em franca evolução, reflete, cada vez mais, a arte e a ciência da enfermagem graças ao esforço e empenho de excelentes enfermeiras clínicas, de pesquisadoras de enfermagem, de outros profissionais e organizações da área ${ }^{(5)}$.

Neste sentido, o presente estudo tem como objetivo compartilhar a experiência vivenciada pelos enfermeiros do DE do HU-USP no primeiro momento do processo de implementação do diagnóstico de enfermagem como etapa do SAE.

Para tanto adota o método descritivo segundo as etapas seqüenciais da realidade concreta da implementação do diagnóstico de enfermagem no HU-USP.

\section{A IMPLEMENTAÇÃO DO DIAGNÓSTICO DE ENFERMAGEM NO SISTEMA DE ASSISTÊNCIA DE ENFERMAGEM}

\section{Cenário Institucional}

O quadro dos profissionais de enfermagem do DE é composto por 650 funcionários, sendo: 1 Diretora de Departamento, 4 Diretoras de Divisão, 1 Diretora de Serviço, 13 Chefes de Seção, 161 Enfermeiros, 54 Técnicos de Enfermagem e 416 Auxiliares de Enfermagem. Estes profissionais estão distribuídos em quatro divisões: Divisão de Enfermagem Cirúrgica (DEC), Divisão de Enfermagem Clínica (DECLI), Divisão de Enfermagem Materno-Infantil (DEMI) e Divisão de Pacientes Externos (DEPE). Essas quatro divisões congregam treze seções e três setores.

O DE agrega ainda o Serviço de Apoio Educacional (SEd), que engloba a Escola de Técnico de Enfermagem e a Educação Continuada. Conta, também, com órgãos de assessoria como a Comissão de Ética e Grupos de Estudos.

\section{A trajetória percorrida}

Como um primeiro momento foi realizado, em Dezembro de 2001, um seminário enfocando a informatização da documentação clínica de enfermagem, com a participação de cerca de $60 \%$ dos enfermeiros em exercício no HU-USP. A proposta desse seminário foi a de sensibilizar os enfermeiros para a necessidade de planejar processos que sustentassem as mudanças para a informatização da documentação do SAE. Ao término do evento o grupo de enfermeiros, comprometeu-se a delinear um plano para a implementação do diagnóstico de enfermagem. 
Em Março de 2002, como segundo momento, foi realizado um curso teórico-prático de 16 horas, ministrado por uma docente da Escola de Enfermagem (EE) da USP com grande experiência no processo de diagnóstico em enfermagem. Contou com a participação da Diretora do DE, das Diretoras de divisão, da Diretora do Serviço de Apoio Educacional, das Chefes de Seção, dos enfermeiros representantes de unidades de enfermagem onde não havia designação de cargo de chefia (Hemodiálise e Endoscopia) e de duas docentes da EEUSP. A proposta deste curso foi a de capacitar os participantes para se tornarem agentes multiplicadores do conteúdo teórico-prático sobre diagnóstico de enfermagem junto a todos os enfermeiros assistenciais do DE.

Após a realização do curso foi criado o Grupo Facilitador para a Implementação do Diagnóstico de Enfermagem (GFIDE), que passou a se reunir, sistematicamente, para discussão e criação de um plano de ação. Foi então concebido um curso para abordar e discutir o diagnóstico em enfermagem, visando a sensibilização dos enfermeiros assistenciais para a sua implementação; enfatizando a importância do diagnóstico para o desenvolvimento do raciocínio clínico do enfermeiro e para a qualidade da assistência de enfermagem; enfocando o diagnóstico como segunda etapa do SAE e estudando a estrutura dos diagnósticos proposta pela North American Nursing Diagnosis Association - NANDA ${ }^{(6)}$.

O I Curso de Diagnóstico de Enfermagem para enfermeiros assistenciais do HU-USP foi realizado em Junho de 2002, durante três dias, com duração total de seis horas. Foram abordados tópicos sobre a introdução do diagnóstico de enfermagem no HU, a importância do diagnóstico como etapa do SAE, a história do diagnóstico em enfermagem, a padronização da linguagem, a estrutura do diagnóstico proposta pela NANDA, as etapas do raciocínio clínico, o estudo de caso para a elaboração de diagnóstico de enfermagem e considerações sobre os benefícios e tendências do diagnóstico na profissão de enfermagem. Além disso, foi apresentado o esboço de um cronograma para a implementação do diagnóstico no SAE para que os participantes pudessem opinar e propor sugestões.

Dos $161(100 \%)$ enfermeiros assistenciais que faziam parte do quadro de pessoal do DE, $142(88,2 \%)$ participaram desse curso. Ao final do mesmo foi solicitado, aos participantes, que preenchessem um impresso de avaliação considerando se o curso havia correspondido às suas expectativas, se havia contribuído para o seu crescimento profissional e se o local, o horário, o conteúdo, a organização e os recursos audiovisuais utilizados haviam sido pertinentes e adequados. Dos participantes que devolveram o impresso de avaliação $113(79,6 \%)$ referiram que o curso propiciou crescimen- to profissional e $84(59,1 \%)$ responderam que correspondeu às suas expectativas. A maioria dos respondentes classificou como bom, o local, o horário, o conteúdo, a organização e os recursos audiovisuais.

É importante ressaltar que durante a realização desse curso pode-se constatar que emergiram sentimentos conflitantes entre os participantes. Alguns demonstraram interesse na nova proposta de trabalho e a grande maioria não tinha conhecimento prévio sobre o conteúdo abordado, referindo sentir-se preocupada com as mudanças que seriam necessárias para a implementação do diagnóstico como etapa do SAE. Outros, ainda, posicionaram-se com resistência à nova proposta afirmando que esta traria sobrecarga de trabalho para o enfermeiro.

Cientes destas manifestações os membros do GFIDE elaboraram um cronograma para a implementação do diagnóstico de enfermagem, utilizando o referencial teórico da NANDA, no SAE do HU-USP. A época considerada adequada para que a maioria das unidades estivesse documentando os diagnósticos de enfermagem nos prontuários foi Janeiro de 2003. Para atingir essa meta estabeleceu-se um plano de trabalho para o $2^{\circ}$ semestre de 2002.

Assim, a partir de Julho de 2002 tiveram início reuniões semanais nos diferentes plantões, coordenadas pela enfermeira chefe de cada unidade, quando os enfermeiros passaram a realizar e apresentar estudos de caso levantando os diagnósticos de enfermagem mais freqüentes nas suas unidades. Nestas reuniões a enfermeira chefe identificava as expectativas do grupo e registrava as propostas sugeridas para a implementação do diagnóstico de enfermagem. A meta estabelecida foi a de que cada enfermeiro realizasse e apresentasse, pelo menos, um estudo de caso completo por mês, em sua respectiva unidade, até a concretização da implementação do diagnóstico de enfermagem. Para viabilizar a realização dos estudos de caso os enfermeiros deveriam se fundamentar no conhecimento orientador de sua prática assistencial bem como nos protocolos existentes na instituição.

Foi elaborado um roteiro onde o enfermeiro deveria registrar breve histórico do paciente, com dados do exame físico e entrevista; listar as características definidoras ou fatores de risco observados; formular possíveis diagnósticos de enfermagem; escolher os diagnósticos de enfermagem definitivos ${ }^{(a)}$ e propor intervenções de enfermagem, a eles correspondentes. Havia ainda um campo para o registro de sugestões e observações acerca do processo diagnóstico.

(a) Entende-se por diagnósticos de enfermagem definitivos aqueles que os enfermeiros, após análise e discussão dos diagnósticos possíveis, consideram como mais adequados para expressar as respostas apresentadas pelos pacientes a problemas de saúde / processos de vida. 
Ainda em Julho de 2002 foi realizado o II Curso de Diagnóstico de Enfermagem para enfermeiros assistenciais do HU-USP, para 15 (9,3\%) enfermeiros que estavam em férias ou em licença, cujo conteúdo era similar ao do primeiro curso, porém, sintetizado para ser ministrado num único dia, com duração de quatro horas abrangendo, assim, o total de 157 (97,5\%) enfermeiros do quadro de pessoal do DE. A partir deste curso o SEd acrescentou conteúdo referente ao diagnóstico de enfermagem no programa de treinamento admissional a fim de iniciar a capacitação dos profissionais da equipe de enfermagem que fossem admitidos.

Em Agosto de 2002 foram realizadas reuniões científicas sobre o Diagnóstico de Enfermagem com os profissionais de nível médio do quadro de pessoal do DE, com a duração média de duas horas, em três horários diferentes para profissionais dos diferentes turnos de trabalho. Estas reuniões tiveram como objetivo apresentar, aos técnicos e auxiliares de enfermagem, a proposta de implementação do diagnóstico de enfermagem no SAE e enfatizar a importância da participação ativa, de cada um, no processo de levantamento de dados e no planejamento da assistência. Chamou atenção a aparente tranqüilidade e receptividade destes profissionais em relação ao diagnóstico de enfermagem e a sua insatisfação por não participar, efetivamente, do planejamento da assistência, sendo relegados a meros executores da prescrição realizada pelo enfermeiro. Este fato mostrou um outro desafio que era o de conscientizar o enfermeiro da importância de envolver a equipe e com ela discutir o plano de cuidados a fim de tornála partícipe e ativa na prestação da assistência.

Em Setembro de 2002 foi realizada uma oficina de trabalho onde cada divisão do DE apresentou um caso com os respectivos diagnósticos de enfermagem elaborados. Participaram deste evento especialistas convidados que discutiram e trocaram experiências sobre o processo diagnóstico adotado em cada caso. Pôde-se observar que os enfermeiros que participaram deste evento demonstravam estar comprometidos com a implementação do diagnóstico no SAE apesar das dificuldades e dúvidas evidenciadas.

Concomitantemente ao desenvolvimento do processo de implementação do diagnóstico de enfermagem e sua melhor inserção no SAE, havia uma proposta da Diretora do DE para a adoção de um novo sistema de trabalho, denominado Primary Nursing. O Primary Nursing é um sistema para a aplicação de serviços de enfermagem constituído por quatro elementos estruturais: alocação e aceitação de responsabilidade individual pela tomada de decisões por uma pessoa; designação de cuidados diários pelo método de casos; comunicação direta pessoa/pessoa e uma pessoa operacionalmente responsável pela qualidade de cuidados administrados a pacientes numa unidade vinte-e-quatrohoras-por-dia, sete dias por semana ${ }^{(7)}$. Em síntese, este sistema prevê a existência de uma equipe de enfermagem responsável pelas intervenções de enfermagem bem como pela avaliação dos resultados da assistência prestada a determi- nados pacientes, durante toda a internação. Foi então realizado outro Workshop, em Outubro, aberto a todos os membros da equipe de enfermagem, relacionando o Primary Nursing com o diagnóstico de enfermagem, buscando-se ressaltar a pertinência deste sistema no contexto do diagnóstico de enfermagem.

Todos estes eventos e a aproximação da implementação do diagnóstico de enfermagem evidenciaram a necessidade, premente, de serem revisadas as normas do SAE a fim de que realmente ocorresse a aproximação da teoria à prática cotidiana dos enfermeiros. Nesse sentido, foi realizada uma oficina de trabalho com os membros do GFIDE quando, partindo de estudos de casos vividos, tiveram inicio as discussões sobre mudanças necessárias nas diferentes etapas do processo de enfermagem. Para tanto, os participantes foram divididos em grupos que ficaram responsáveis pela discussão e análise de uma etapa específica do SAE, propondo as mudanças que considerassem importantes e apresentandoas ao GFIDE. Desde o início desta oficina os membros do GFIDE tiveram a clareza da necessidade de participação dos enfermeiros assistenciais nas discussões para revisão do $\mathrm{SAE}$, principalmente, porque estes queixavam-se, freqüentemente, do tempo que era gasto para a sua operacionalização. No entanto, pelas dificuldades que acompanham todo processo participativo, considerou-se mais adequado que, a princípio, fossem elaboradas algumas propostas para posterior apresentação aos enfermeiros assistenciais. Dessa forma, os enfermeiros assistenciais poderiam posicionarse a respeito destas propostas e sugerir outras participando, ativamente, das mudanças mais pertinentes. Decidiu-se, então, que seriam realizadas visitas a outras instituições que já tivessem o diagnóstico de enfermagem implementado com a finalidade de conhecer sua operacionalização e compartilhar das experiências de outros enfermeiros neste processo.

Após visita a duas instituições, os membros do GFIDE começaram a estruturar um instrumento provisório para o registro dos diagnósticos de enfermagem no SAE e continuaram, paralelamente, a revisar as normas do SAE com o propósito de agilizar estas atividades e apresentá-las aos enfermeiros assistenciais para que juntos buscassem consenso.

Com vistas à facilitação da criação de um instrumento que se adequasse à filosofia do DE e à realidade dos enfermeiros do HU-USP, optou-se pela realização de um estudo para o levantamento de todos os diagnósticos de enfermagem apresentados pelos pacientes nas diversas unidades. No período de um mês, 13/01 a 13/02/2003, os enfermeiros realizaram o levantamento dos diagnósticos de enfermagem apresentados pelos pacientes sob seus cuidados. Para tanto utilizaram a mesma metodologia empregada nos estudos de caso. Ao final do período estabelecido, a chefe de sessão encaminhou todos os diagnósticos registrados pelos enfermeiros de sua unidade ao SEd para a construção de um banco de dados. 
Tendo início a construção do banco de dados percebeuse que esta etapa demandaria um tempo maior do que o previsto devido à complexidade da conversão dos dados obtidos para a linguagem informatizada. Vale registrar que para a criação desse banco de dados havia necessidade de alguém que possuísse ampla experiência em informática e conhecimento do processo diagnóstico em enfermagem, sendo indicada uma enfermeira do SEd que, em virtude de outras atribuições, não pode ser designada somente para esta atividade.

Em Abril de 2003, após uma reunião do GFIDE, decidiu-se que os enfermeiros deveriam prosseguir com o levantamento dos diagnósticos de enfermagem apresentados por dois pacientes sob seus cuidados, por semana, com a finalidade de estimular a manutenção e desenvolvimento da atividade diagnóstica até que se viabilizasse a implementação do diagnóstico de enfermagem no SAE. Ao final de cada mês as chefes de seção encaminhavam os diagnósticos elaborados pelos enfermeiros de sua unidade para o SEd, para que fossem agregados ao banco de dados, em construção. Concomitantemente a essas atividades, foi propiciado a alguns enfermeiros, representantes de diferentes unidades do DE, a participação em eventos relacionados ao diagnóstico de enfermagem.

As discussões sobre as mudanças necessárias no SAE prosseguiam principalmente em relação à evolução e anotação de enfermagem. Avaliando-se vários prontuários percebia-se que, em algumas unidades, as evoluções de enfermagem eram muito extensas, demandando um tempo maior da enfermeira para sua realização. Na maioria das unidades, as evoluções de enfermagem repetiam informações contidas nos demais impressos tais como gráficos, resultados de exames, anotação de enfermagem e evolução médica. Havia ainda unidades onde as anotações de enfermagem eram muito semelhantes à evolução de enfermagem. Após a conclusão da revisão das normas para o registro da evolução e anotação de enfermagem, foi decidido que as mesmas deveriam ser apresentadas aos membros da equipe de enfermagem.

Em Maio de 2003, a Diretora do DE realizou reuniões com os membros da equipe de enfermagem quando foram compartilhadas as propostas de mudanças nas normas do SAE. Durante as reuniões várias pessoas manifestaram sua aprovação a estas propostas referindo que estas direcionariam, melhor, os registros de enfermagem. Após as reuniões decidiu-se que as normas revisadas do SAE vigorariam a partir de Junho de 2003. Paralelamente a este movimento, alguns membros do GFIDE prosseguiram com a discussão da criação de um instrumento para o registro dos diagnósticos de enfermagem. Havia a intenção de listar, nesse instrumento, os diagnósticos de enfermagem mais freqüentes em cada unidade, com as respectivas intervenções de enfermagem, deixando-se um espaço para o acréscimo de outros diagnósticos e intervenções que a enfermeira julgasse necessário. Percebeu-se que este instrumento deveria contemplar as particularidades de cada unidade, sendo importante a realização de um teste piloto, em uma unidade onde os diagnósticos de enfermagem, elaborados pelos enfermeiros, já estivessem inseridos no banco de dados.

Nessa mesma época os diagnósticos de enfermagem das unidades de Clínica Médica, Pronto Socorro Infantil, Unidade de Terapia Intensiva Adulto e Berçário já estavam contidos no banco de dados. Ao analisar-se as características destas unidades optou-se pela realização do projeto piloto na unidade de Clínica Médica que, por ser uma unidade de internação de pacientes adultos, poderia ter os resultados replicados em outras unidades afins.

A avaliação desta etapa inicial do processo de implementação do diagnóstico de enfermagem evidencia a importância da realização do levantamento de necessidades e a mobilização de recursos para o atendimento dessas processo participativo adotado, nessa trajetória, mostra que sua implantação exige de todos que dela compartilham necessidades tanto de ordem técnica, disponibilizando recursos estruturais e tempo para propiciar a capacitação das pessoas envolvidas, como de ordem ético-política, promovendo a participação efetiva das pessoas envolvidas com a valorização do seu conhecimento e potencial criativo. Esses elementos subsidiaram a avaliação positiva do início deste processo, em construção, e têm permitido a sua continuidade que se pretende divulgar posteriormente.

\section{CONSIDERAÇÕES FINAIS}

Apesar das dificuldades e incertezas que permearam a fase inicial do processo de implementação do diagnóstico de enfermagem, pode-se perceber a preocupação dos enfermeiros do DE do HU-USP em fundamentar, com referencial teórico, a experiência vivenciada na prática contribuindo, assim, para a construção desse conhecimento em enfermagem. A trajetória descrita poderá subsidiar outras instituições que queiram implementar o diagnóstico de enfermagem.

O processo participativo adotado, nessa trajetória, mostra que exige tempo e determinação de todos que dela compartilham. Entretanto, pela experiência vivenciada, podese resgatar também, que o resultado se traduz em crescimento individual e, conseqüentemente, em efetivo produto coletivo. Se por um lado há a necessidade de se promover a capacitação dos enfermeiros, exigindo deles esforço e compromisso, por outro lado, a instituição tem que propiciar todas as condições para que isto aconteça. 


\section{REFERÊNCIAS}

(1) Horta WA. Processo de enfermagem. São Paulo: EPU/EDUSP; 1979.

(2) Orem DE. Nursing concepts of pratice. New York: McGrawHill; 1985.

(3) Cruz DALM. A inserção do diagnóstico de enfermagem no processo assistencial. In: Cianciarullo TI, Gualda DMR, Melleiro MM, Anabuki, MH. Sistema de assistência de enfermagem: evolução e tendências. São Paulo: Ícone; 2001. p. 63- 84.

(4) Rossi LA, Dalri MCB. Processo de enfermagem: análise e propostas de reformulação segundo modelo conceitual de Horta e Taxonomia I dos diagnósticos de enfermagem da NANDA. Rev Esc Enferm USP. 1993;27(3):328-58.
(5) Carpenito LJ. Diagnósticos de enfermagem: aplicação à prática clínica. Trad. De Ana Thorell. $8^{\mathrm{a}}$ ed. Porto Alegre: Artmed; 2002.

(6) North American Nursing Diagnosis Association (NANDA). Diagnósticos de enfermagem da NANDA: definições e classificação. 1999-2000. Porto Alegre: Artes Médicas Sul; 2000.

(7) Manthey, MER. A prática do Primary Nursing (Enfermeira Principal). Boston: Blackwell; 1980. Elementos de Primary Nursing; p. 30-44. 